\title{
Acupuncture in Shoulder Pain and Functional Impairment After Neck Dissection: A Prospective Randomized Pilot Study
}

\author{
Alberto Deganello, MD, PhD; Nir Battat, MD; Enrico Muratori, MD; Glauco Cristofaro, MD; \\ Ana Buongiorno, MD; Giuditta Mannelli, MD; Mario Picconi, MD; Rita Giachetti, MD; \\ Giulia Borsotti, MD; Oreste Gallo, MD
}

\begin{abstract}
Objectives/Hypothesis: The efficacy of conventional physiotherapy and antiinflammatory/analgesic drugs in the management of shoulder pain and functional disability following neck dissection is often disappointing. Acupuncture is a safe and well-tolerated method. We report the results regarding our pilot trial of acupuncture versus conventional care in the management of postoperative shoulder pain and dysfunction after neck dissection.

Study Design: Pilot study.

Methods: Patients at a tertiary university center with chronic pain or dysfunction attributed to neck dissection were randomly assigned to either weekly acupuncture or usual care (eg., physical therapy, analgesia, and/or antiinflammatory drugs) for 5 consecutive weeks.

The Constant-Murley score, a composite measure of pain, function, and activities of daily living, was the primary outcome measure. As secondary end point, The Neck Dissection Impairment Index (NDII) was used to quantify site-specific, selfreported quality of life (QOL).

Results: After randomization, 48 patients completed the study (23 and 25 patients on acupuncture and control arms, respectively). Constant-Murley scores improved more in the acupuncture group (gain difference between groups $13.6, P<$ 0.01 ), a statistically significant improvement in site-specific QOL was also recorded at NDII (gain difference between groups 11.5, $P<0.01)$.

Conclusion: Acupuncture is safe and effective; it should be introduced and offered to patients suffering from neck pain and dysfunction related to neck dissection.

Key Words: Acupuncture, neck dissection, neck disability, neck pain, shoulder syndrome, shoulder dysfunction.

Level of Evidence: Prospective randomized pilot study (2b).
\end{abstract}

Laryngoscope, 00:000-000, 2016

\section{INTRODUCTION}

Neck dissection remains a crucial procedure in the management of head and neck tumors. In order to guarantee adequate regional control while minimizing postoperative morbidity, the surgical technique has evolved over time with the intent of preserving nonlymphatic structures and neck levels at lowest risk for occult disease. ${ }^{1}$ Therefore, indications and extensions of the procedure are constantly refined. ${ }^{2-4}$ Although in neck dissection procedures the preservation of the spinal accessory nerve currently is more common, shoulder dys-

From the 1st Clinic of Otolaryngology-Head and Neck Surgery, Department of Surgery and Translational Medicine (A.D., N.B., E.M., G.C., A.B., G.M., O.G.); the Department of Anaesthesiology (R.G., G.B.), University of Florence, Florence; and the Acupuncture, Sinergie Center (M.P.), Florence, Italy.

Editor's Note: This Manuscript was accepted for publication January 20, 2016.

The authors have no funding, financial relationships, or conflicts of interest to disclose.

Send correspondence to Alberto Deganello, 1st Clinic of Otolaryngology-Head and Neck Surgery, Department of Surgery and Translational Medicine, University of Florence, Largo Brambilla 3, 50134 Florence, Italy. E-mail: adeganello@hotmail.com

DOI: 10.1002/lary.25921 function and pain occurs in $20 \%$ up to $60 \%$ of the patients. $^{5-7}$

Physiotherapy and analgesic or antiinflammatory drugs are commonly prescribed; however, their effectiveness is not supported by strong evidence in literature. Also, from our clinical experience, it has emerged that the efficacy of conventional treatments was often disappointing or incomplete. Recently, two controlled trials showed the superiority of both progressive resistance exercise $^{8}$ and acupuncture ${ }^{9}$ over standard treatment in patients experiencing shoulder pain and dysfunction after neck dissection.

Acupuncture is a safe and well-tolerated treatment, and the understanding regarding the physiologic basis of its efficacy is growing. The main therapeutic effects of needling are achieved by means of stimulation of the nervous system ${ }^{10}$ through local antidromic axon reflexes, releasing neuropeptides ${ }^{11}$ and increasing local nutritive blood flow. ${ }^{12}$ In both the spinal cord and brain, it causes release of the opioid peptides and serotonin, ${ }^{13}$ as documented by imaging studies with functional magnetic resonance imaging and positron emission tomography, which provided good evidence of the effects on various brain areas involved in pain control. ${ }^{14-16}$ The clinical effects on musculoskeletal pain are explained by inhibition of the 
nociceptive pathway at the dorsal horn, through activation of the descending inhibitory pathways and possibly by local or segmental effects on myofascial trigger points. ${ }^{13,17,18}$

Clinical randomized trials demonstrated the efficacy of acupuncture for neck and shoulder pain, attributed to etiologies not related to malignancy or its treatment. ${ }^{19-24}$ Moreover, a recent case report showed its efficacy in the management of first bite syndrome following surgery of the parapharyngeal space..$^{25}$

We report the results of our pilot study of acupuncture versus conventional care in the management of postoperative shoulder pain and dysfunction after neck dissection. The primary goal of the study was to determine the effectiveness of acupuncture in reducing pain and improving shoulder function using the Constant Murley Score (CMS). ${ }^{26}$ The secondary goal was to evaluate the impact of acupuncture on the site-specific, selfreported quality of life (QOL) in these patients as measured by the Neck Dissection Impairment Index (NDII). ${ }^{27}$

\section{MATERIALS AND METHODS}

\section{Experimental Design}

We conducted a prospective pilot study in which the efficacy of acupuncture versus conventional care was evaluated using two validated tools designed specifically for the assessment of the shoulder: the CMS and the NDII.

Eligible participants were stratified by age, sex, type of neck dissection, and baseline NDII score to control for potential differences in QOL and recovery. Patients were randomly assigned to acupuncture or to usual care by means of a secure computerized database, ensuring full allocation concealment.

Approval of the study protocol was received from the institutional review board.

\section{Patient Selection Criteria}

At the Otolaryngology-Head and Neck Surgery Department of the University of Florence, 169 patients who were admitted to routine follow-up outpatient consultation in the last 6 months of 2014, and who received a neck dissection between January 2010 and December 2014, were enrolled for this study. The NDII questionnaire was administered to all 169 potentially eligible patients during clinical follow-up. From this population, 74 patients with shoulder dysfunction and/or pain in at least one of the upper limbs attributed to neck dissection, scoring below 90 at NDII, were further evaluated for inclusion and exclusion criteria regarding trial recruitment (Figure 1).

Inclusion criteria included the availability to follow acupuncture for 5 consecutive weeks, and for a follow-up evaluation at least 1 month after the last session; unilateral neck dissection; time frame of at least 1 month after previous neck dissection or adjuvant radiotherapy (RT)/chemoradiation CRT and issuance of a signed informed consent regarding participation in this study.

Exclusion criteria were a history of rehabilitation physiotherapy or acupuncture performed 2 months previous to routine follow-up; bilateral neck dissection; evidence of locoregional/distant failure; evidence of second primaries.

Thirteen patients did not meet eligibility criteria and an additional eight patients refused to participate, which resulted in a cohort of 53 patients undergoing randomization.

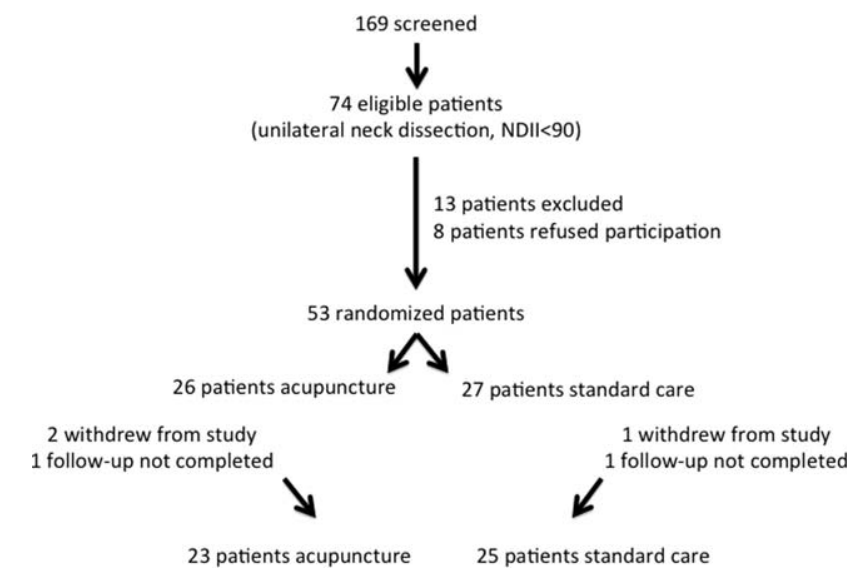

Fig. 1. Study flowchart.

For all patients, the following data were obtained: demographic data; histology; tumor-node-metastasis (TNM) stage; type of surgery at primary tumor; type of neck dissection; type of adjuvant treatment RT/CRT; and baseline outcome of the assessments.

\section{Assessment Methodology}

All patients were tested in both arms by means of the CMS and the NDII. Assessments were performed before randomization, directly after completed treatment, and 1 month after the last session. The CMS, which integrates scores of subjective and objective parameters, provided the primary outcome measure. This validated clinical assessment of shoulder function has established utility and accuracy across all diseases that affect the shoulder. Moreover, this widely used clinical test is an accurate and sensitive measure of shoulder function, detecting even subtle changes. The four subscales that compose the CMS are pain (15 points), activities of daily living (20 points), pain-free range of motion using a universal goniometer according to standardized procedures (40 points), and pain-free ability to generate muscle strength measured in kilograms (25 points). ${ }^{18}$ The final score ranges from 0 up to 100 with higher scores representing better outcome. The evaluation of the CMS was performed by a physician who was blinded of patient's treatment allocation. This scale proves to have low levels of operator-related errors, shows good responsiveness, and was successfully applied in a prior randomized trial regarding acupuncture for shoulder pain. , $^{9,29}$

The secondary endpoint, the impact of treatment-specific QOL, was evaluated using the NDII, which is a reliable instrument to assess this purpose. The NDII is a subjective, selfreported, validated questionnaire that includes 10 individual items. The degree of discomfort per item was scored from 1 to 5 ; a score of 5 represents good QOL related to neck dissection (5: no discomfort at all, 4: a little discomfort, 3: a moderate amount of discomfort, 2: quite a bit of discomfort, 1: a lot of discomfort). Subsequently, the NDII scores were transformed to a 0 to 100 point scale $[($ raw score -10$) / 40] \times 100$, with higher scores representing better QOL.

\section{Treatment}

Acupuncture: all 26 patients underwent a weekly session of acupuncture that lasted between 30 and 45 minutes for 5 consecutive weeks; 23 patients completed follow-up evaluation (30 days after the last session). The acupuncture procedure was 
performed according to protocol of an acupuncturist with at least 10 years of experience, overseeing and guiding physician acupuncturists in conducting the sessions (M.P.). Acupuncture needles were placed according to traditional Chinese medicine acupuncture technique-at a depth of $0.6 \mathrm{~cm}$ to $1.2 \mathrm{~cm}$ in standard points (local and distant), and customized points for each patient based on the patients primary pain location-with the intent of optimizing efficacy while permitting reproducibility. The standard distal points indicated for the treatment of shoulder pain dysfunction were LI-4, SI-3, SI-6, GB-34, TB-14, TB-6, LI-15, GB-21, Shenmen (Au), and Luozhen (Ex) (Figure 2 ). LI-4 is indicated for pain of the head and neck region; and SI-6, SI-3, and GB-34 are indicated to treat low back pain and cervical-scapular pain. Luozhen is located outside of the meridians and is indicated to treat stiffness of the neck and shoulder. The auricular Shemen point is traditionally used to relieve pain. Custom points (front, middle, and back) were chosen based on the primary location of the pain. Sterile, stainlesssteel, single-use, filiform needles (20-30 mm in width) were used in this study.

No formal limitations regarding the use of analgesic drugs were prescribed, whereas rehabilitation physiotherapy was prohibited during the period of acupuncture.

The acupuncture therapist and examiners monitored adherence and all adverse events of therapy during the trial.

Conventional Care: All 27 patients in this group were instructed by a professional physiotherapist to follow 5 consecutive weeks of current standard therapy for shoulder dysfunction and pain. Patients carried out daily physical exercises of the upper limb autonomously, which included active and passive stretching, postural exercises, and basic strengthening exercises with light weights (not superior than $5 \mathrm{~kg}$ ) or elastic resistance bands. These patients were allowed to use analgesic/antiinflammatory drugs at need and attended weekly consultations with the physiotherapist, who oversaw the adherence to the prescribed protocol; 25 patients completed follow-up evaluation.

\section{Statistical Analysis}

All data were analysed using Stata/SE 12.0 (StataCorp, College Station, TX). Categorical data were evaluated using the Fisher exact test. Continuous data were assessed by Student $t$ test. Differences in mean values between groups were tested with Wilcoxon-Mann-Whitney test. Probability values $<0.05$ were considered statistically significant.

\section{RESULTS}

\section{Study Population}

A total of 169 patients were initially enrolled to be tested with baseline NDII; 44 patients received bilateral neck dissection accounting for 213 neck dissections: two radical neck dissections (RND), 87 modified radical neck dissections (mRND), 108 selective neck dissections (SND), and 16 superselective neck dissections (levels IIa-III). Seventy-four patients were preselected (none of them had a previous bilateral neck dissection) who scored at least $10 \%$ of impairment at the NDII, with a final score $<90$ points. In this population, 45 patients were males (61\%); the medium age was 64.5 years (range: 34-90, median: 68). Eventually, 13 patients did not meet inclusion criteria and 8 patients refused participation, resulting in a population of 53 patients undergoing randomization. The randomization assigned 26

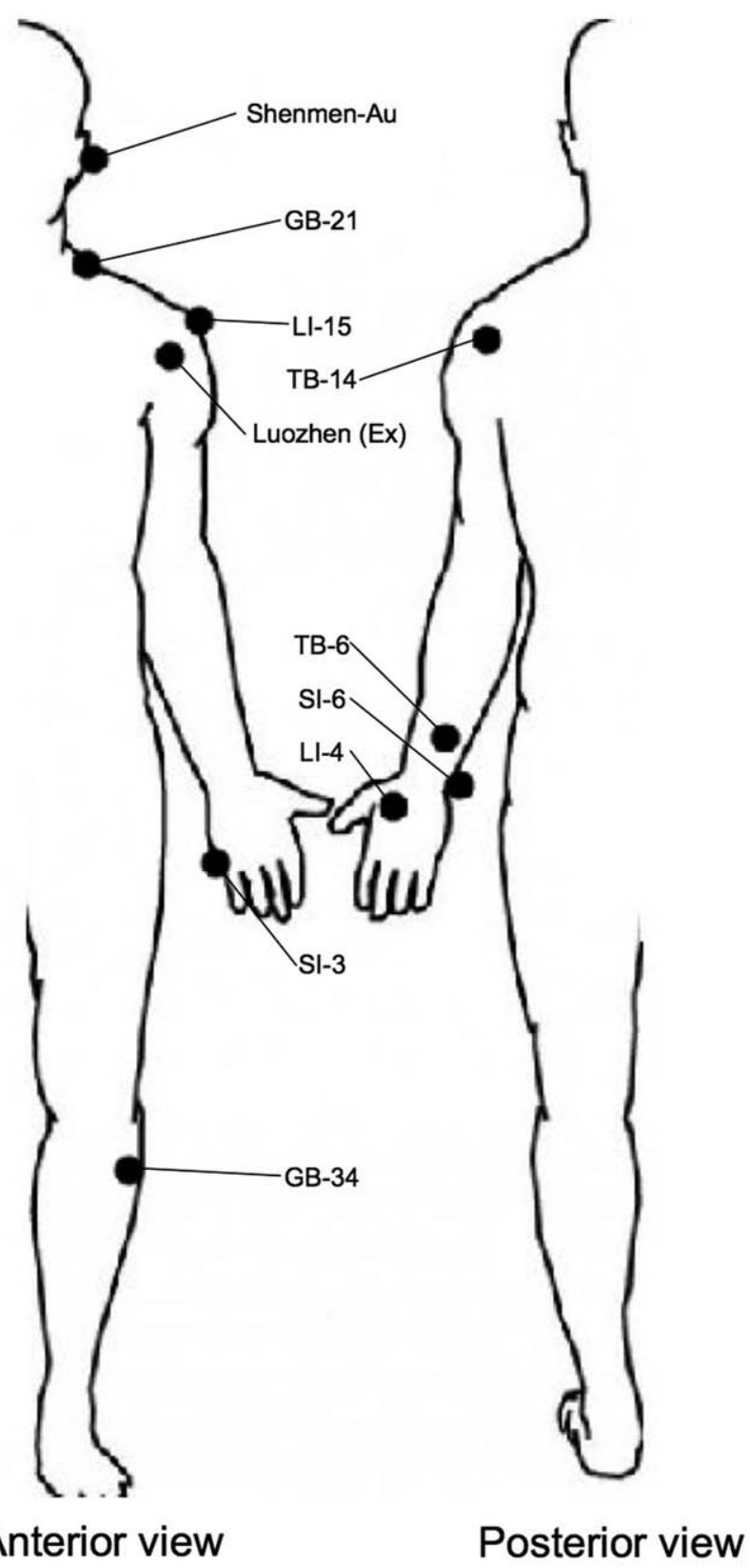

Fig. 2. Body diagram depicting acupuncture sites location.

patients to acupuncture and 27 patients to usual care; however, the definitive study population contained 48 patients because 23 patients completed the study in acupuncture and 25 patients in the conventional care group. Table I shows the clinical characteristics of the studied population, with no significant differences between the two groups (of some note: all mRNDs were type III, with preservation of the spinal accessory nerve, internal jugular vein, and sternocleidomastoid muscle).

Figure 3 displays the results measured after completed treatment, showing that acupuncture was significantly superior over standard care. The evaluation of the primary endpoint with CMS revealed that acupuncture patients had a mean score improvement of 15.7 (standard deviation [SD] 9.2) points over 2.1 points in 


\begin{tabular}{lccc}
\hline \multicolumn{4}{c}{ TABLE I. } \\
& Study Population. & \\
\hline & $\begin{array}{c}\text { Acupuncture } \\
\text { Group, } \mathrm{n}(\%)\end{array}$ & $\begin{array}{c}\text { Standard Care, } \\
\mathrm{n}(\%)\end{array}$ & $\begin{array}{c}P \text { Vauncture } \\
\text { vs. Control }\end{array}$ \\
\hline Patients & $23(47.9) ;$ & $25(52.1) ;$ & \\
Age, yr, mean \pm SD & $60.1 \pm 10.7$ & $63.4 \pm 9.2$ & $0.26^{\star}$ \\
Sex, male & $15(65.2)$ & $17(68)$ & $0.84^{\dagger}$ \\
Neck Dissection Type & & & $0.42^{\ddagger}$ \\
SSND & $1(3.7)$ & $1(3.4)$ & \\
SND & $11(40.7)$ & $9(31.0)$ & \\
mRND & $14(51.8)$ & $18(62.0)$ & \\
RND & $1(3.7)$ & $1(3.4)$ & $0.75^{\ddagger}$ \\
Histotype & & & \\
Squamous cell & $19(82.6)$ & $20(80.0)$ & \\
carcinoma & $2(8.7)$ & $3(12.0)$ & \\
Melanoma & $2(8.7)$ & $2(8.0)$ & \\
Thyroid carcinoma & & & $0.75^{\ddagger}$ \\
Adjuvant Treatment & & $9(36.0)$ & \\
None & $9(39.1)$ & $9(36.0)$ & \\
RT & $7(30.4)$ & $7(28.0)$ & \\
CRT & $7(30.4)$ & & \\
\hline \hline
\end{tabular}

\footnotetext{
${ }^{*}$ Student $t$ test.

${ }^{\dagger}$ Fisher exact test.

${ }^{\mp} \chi^{2}$ test.

CRT $=$ chemoradiation; $\mathrm{mRND}=$ modified radical neck dissections; $\mathrm{RND}=$ radical neck dissections; $\mathrm{RT}=$ radiotherapy; $\mathrm{SD}=$ standard deviation; SND = selective neck dissections ; SSND = superselective neck dissections; $y r=$ year.
}

standard care $(P<0.01)$; this difference of 13.6 points was maintained after re-measurements at 1 month follow-up.

Concerning the secondary endpoint, patients receiving acupuncture showed a consistent improvement in site-specific QOL as measured by means of the NDII, with a mean score of 16.1 points over 4.6 points in standard care $(P<0.01)$. Additionally, this difference of 11.5 points was consistent after re-assessment at 1 month follow-up. No serious adverse events were attributed to acupuncture. Twelve minor events were noted in this study, which mainly included minor bruising or bleeding caused by needle positioning and constitutional symptoms.

After the study was closed, long-term (range 4-10 months) follow-up data were obtained in 13 out of 23 patients of the acupuncture group. A further improvement regarding the CMS was recorded, with a new mean score of 83.4 (SD 18.6) and a further average gain of 16.8 points over the score recorded by the same patients after 1 month follow-up. In these patients, the NDII score also further improved, with an average gain of 5.5 points over the 1-month follow-up score. The discordance between this mean gain and the results recorded at CMS is explained by the fact that in one patient the CMS recorded a further gain of 2 points; however, the subjective score at NDII dropped 30 points.

\section{DISCUSSION}

In this study, the inclusion criteria were based on neck impairment regardless of the type of neck dissection; in fact, our aim was to quantify the iatrogenic postoperative impairment and see if we could offer a valid treatment regardless of the type of previous surgery. However, we tried to minimize possible randomization related mismatches between the two groups by stratifying by age, sex, type of neck dissection, and baseline NDII score. We stratified by type of neck dissection and degree of neck impairment because we assumed that less extensive neck surgery might leave the patient with more improvement potentials; the randomization respected these requirements.

Our study is in complete agreement with the first pioneer study by Pfister et al., ${ }^{9}$ in which the authors recorded a mean gain of 13.9 points at CMS after acupuncture treatment.

In our study, the mean postacupuncture gain regarding the CMS was 14.5 points. Additionally, an important finding concerning our primary endpoint was that an objective improvement in every single patient receiving acupuncture could be assessed, with four patients who showed a complete recovery (scoring $100 \%$ at CMS after treatment). However, it must be noted that these four patients had a pretreatment score $(74,79,76$, 59 , respectively) above the mean value of the group (55.6). This suggests that physicians should not underestimate minimal shoulder complaints reported by patients. Those with better baseline performances are more prone to achieve complete functional restoration with acupuncture, whereas unfortunately this was not the case for the standard care group.

The analysis regarding the range of motion before and after acupuncture showed that $78 \%$ of the patients experienced an increase of more than 30 degrees both in abduction and anterior lift of the arm. Furthermore, in 22 out of 23 patients, an increase of muscular strength in abduction without pain was measured, with a mean

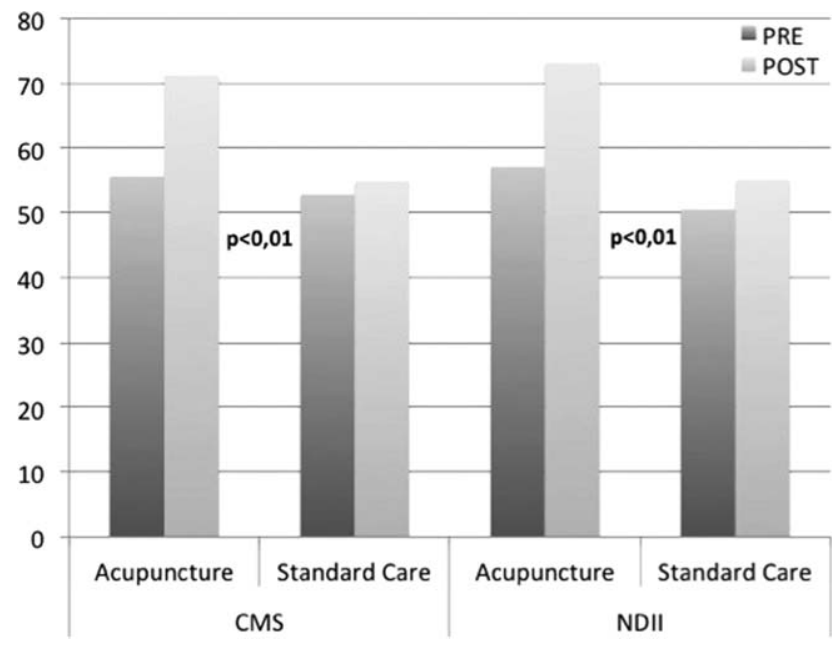

Fig. 3. Results after 1-month follow-up. CMS = Constant-Murley score; NDII = Neck Dissection Impairment Index. 


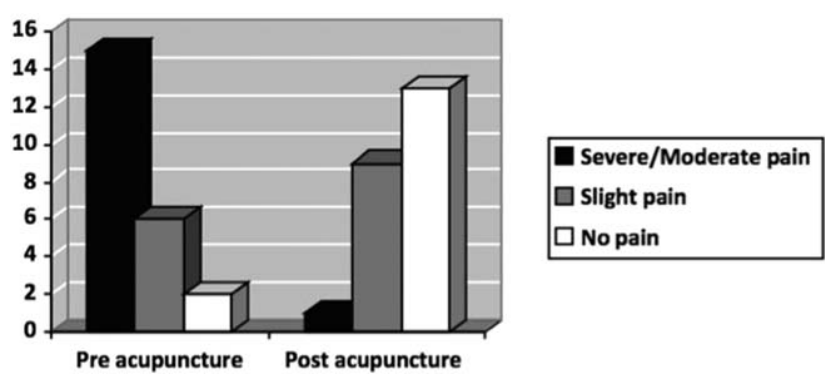

Fig. 4. Pre- and post-acupuncture pain at 1-month follow-up.

increase of 3.7 kilograms (range 1-8). At follow-up consultation 1 month after the last session, the recorded mean value at CMS was $74.8 \%$ (SD 19.5), which means that the beneficial objective effects of acupuncture were not only persistent in time but also were able to foster a small additional improvement. This data was confirmed by the results recorded in 13 acupuncture patients retested between 4 and 10 months after the study; in all these patients, we recorded a further objective improvement at CMS, with an additional mean gain of 16.8 points.

Additionally, analysis of the secondary endpoint showed the efficacy of acupuncture. In this series, six patients out of 23 reported a complete subjective recovery after treatment, with a NDII above $90 \%$. However, two patients had no subjective improvement, although they scored small objective improvements at CMS. The real subjective impact of acupuncture was focused on pain control, with 13 patients $(56.5 \%)$ reporting to be completely free of pain after treatment, and none of them using analgesic drugs anymore (Figure 4).

At 1 month follow-up consultation, the results were consistent with those reported directly after completed treatment. The NDII results at long-term follow-up showed a further improvement over the scores recorded at 1-month follow-up. Of note, the six patients reporting a complete subjective recovery after treatment refused to be re-tested after a few months, claiming a persistent complete wellness. For 12 out of the 13 re-tested patients, a further mean improvement of 13.8 points (SD 7) at NDII was recorded. For the remaining patient, for whom a further gain of 2 points was recorded at CMS, the NDII score dropped of 30 points as compared to the 1 month post-treatment evaluation. The patient claimed that he had to be treated again with acupuncture for free.

Our post-acupuncture scores at NDII are superior to those reported by McNeely et al. ${ }^{8}$ in their randomized controlled trial comparing the effects of progressive resistance exercise training (PRET) versus results obtained with conventional physiotherapy. Progressive resistance exercise training showed superior results regarding the improvement of muscular strength in comparison with our results using acupuncture. Mainly, this might be explained by the indirect beneficial effect that acupuncture has on muscle strength by reducing pain and inflammation versus the direct effects exerted by PRET. Because acupuncture and PRET both showed superior efficacy over standard care in reducing shoulder pain and improving shoulder function for neck dissection patients, and because their mechanisms of action are complementary, we think that it would be wise to combine these methods in a single protocol using acupuncture and PRET together in order to find out if their proven beneficial effects on patients could be summed rather than overlapped.

Analysis of our results showed no correlations between results and tumor histology, type of surgery at primary tumor, TNM stage, and adjuvant CT/RT. Better CMS improvements were recorded in patients receiving acupuncture within 1 year after cancer treatment, and in patients with previous SND (17.2, SD 9.4) over mRND (12.9, SD 9.5). Surprisingly, treatment with acupuncture achieved an objective improvement of 11 points at CMS, even in patients submitted to RND and postoperative CRT. The only patient who showed a minimal improvement at CMS (2 points, from 19 to 21) and no improvement at NDII received previous total thyroidectomy with $\mathrm{mRND}$, followed by adjuvant radioiodine therapy for a pT4aN1b papillary thyroid carcinoma 24 months before acupuncture treatment.

\section{CONCLUSION}

Based on our results and experience, acupuncture proved to be a promising and safe treatment for the management of pain and functional impairment of the shoulder after neck dissection.

Further prospective studies with longer follow-up data should be obtained in order to assess the efficacy and the consistency of acupuncture overtime.

\section{BIBLIOGRAPHY}

1. Gallo O, Deganello A, Scala J, et al. Evolution of elective neck dissection in N0 laryngeal cancer. Acta Otorhinolaryngol Ital 2006;26:335-344.

2. Deganello A, Meccariello G, Bini B, et al. Is elective neck dissection necessary in cases of laryngeal recurrence after previous radiotherapy for early glottic cancer? J Laryngol Otol 2014;128:1089-1094.

3. Deganello A, Gitti G, Meccariello G, et al. Effectiveness and pitfalls of elective neck dissection in N0 laryngeal cancer. Acta Otorhinolaryngol Ital 2011;31:216-221.

4. Sarno A, Bocciolini C, Deganello A, et al. Does unnecessary elective neck treatment affect the prognosis of N0 laryngeal cancer patients? Acta Otorhinolaryngol Ital 2004;124:980-985.

5. Dijkstra PU, van Wilgen PC, Buijs RP, et al. Incidence of shoulder pain after neck dissection: a clinical explorative study for risk factors. Head Neck 2001;23:947-53.

6. Nahum AM, Mullally W, Marmor L. A syndrome resulting from radical neck dissection. Arch Otolaryngol 1961;74:424-428.

7. Cappiello J, Piazza C, Nicolai P. The spinal accessory nerve in head and neck surgery. Curr Opin Otolaryngol Head Neck Surg 2007;15:107-111.

8. McNeely ML, Parliament MB, Seikaly H, et al. Effect of exercise on upper extremity pain and dysfunction in head and neck cancer survivors: a randomized controlled trial. Cancer 2008;113:214-222.

9. Pfister D, Cassileth B, Deng G, et al. Acupuncture for pain and dysfunction after neck dissection: results of a randomized controlled trial. J Clin Oncol 2010;28:2565-2570.

10. Zhao ZQ. Neural mechanism underlying acupuncture analgesia. Prog Neurobiol 2008;85:355-375.

11. Dawidson I, Angmar-Mansson B, Blom M, et al. The influence of sensory stimulation (acupuncture) on the release of neuropeptides in the saliva of healthy subjects. Life Sci 1998;63:659-674.

12. Sandberg M, Lundeberg T, Lindberg LG, et al. Effects of acupuncture on skin and muscle blood flow in healthy subjects. Eur J Appl Physiol 2003;90:114-119.

13. Han JS, Terenius L. Neurochemical basis of acupuncture analgesia. Annu Rev Pharmacol Toxicol 1982;22:193-220.

14. Hui KK, Liu J, Makris N, et al. Acupuncture modulates the limbic system and subcortical gray structures of the human brain: evidence from fMRI studies in normal subjects. Hum Brain Mapp 2000;9:13-25. 
15. Dhond RP, Yeh C, Park K, et al. Acupuncture modulates resting state connectivity in default and sensorimotor brain networks. Pain 2008;136: 407-418.

16. Pariente J, White P, Frackowiak RS, et al. Expectancy and belief modulate the neuronal substrates of pain treated by acupuncture. Neuroimage 2005;25:1161-1167.

17. Sandkuhler J. Learning and memory in pain pathways. Pain 2000;88:113118.

18. Staud R, Price DD. Mechanisms of acupuncture analgesia for clinical and experimental pain. Expert Rev Neurother 2006;6:661-667.

19. Kleinhenz J, Streitberger K, Windeler J, et al. Randomized clinical trial comparing the effects of acupuncture and a newly designed placebo needle in rotator cuff tendonitis. Pain 1999;83:235-241.

20. Sun KO, Chan KC, Lo SL, et al. Acupuncture for frozen shoulder. Hong Kong Med J 2001;7:381-391.

21. Ceccheerelli F, Bordin M, Gagliardi G, et al. Comparison between superficial and deep acupuncture in the treatment of shoulder's myofascial pain: a randomized and controlled study. Acupunct Electrother Res 2001; 26:229-238.

22. Vickers AJ. Acupuncture for treatment of chronic neck pain: reanalysis of data suggest that effect is not a placebo effect. BMJ 2001;323:1306.
23. Irnich D, Behrens N, Molzen H, et al. Randomised trial of acupuncture compared with conventional massage and ÓshamÓ laser acupuncture for treatment of chronic neck pain. BMJ 2001;322:1574-1578.

24. Irnich D, Behrens N, Gleditsch J, et al. Immediate effects of dry needling and acupuncture at distant points in chronic neck pain: result of a randomized, double-blind, sham-controlled crossover trial. Pain 2002;99:83-89.

25. Fiorini FR, Santoro R, Cristofaro G, et al Potential use of acupuncture in the treatment of first bite syndrome. Am J Otolaryngol 201;36:484-487.

26. Costant CR, Murley AH. A clinical method of functional assessment of the shoulder. Clin Orthop 1987;214:160-164.

27. Taylor RJ, Chepeha JC, Teknos TN, et al. Development and validation of the neck dissection impairment index: a quality of life measure. Arch Otolaryngol Head Neck Surg 2002;128:44-49.

28. Skutek M, Fremerey RW, Zeichen J, et al. Outcome analysis following open rotator cuff repair: early effectiveness validated using four different shoulder assessment scales. Arch Orthop Trauma Surg 2000;120: 432-436.

29. O'Connor DA, Chipchase LS, Tomlinson J, et al. Arthroscopic subacromial decompression: responsiveness of disease-specific and health-related quality of life outcome measures. Arthroscopy 1999;15:836-840. 